\title{
Erratum
}

\section{Case Report 225}

Skeletal Radiology (1983) 9:223-225

Hector Ramirez, Jr., M.D., Major, MC, USA ${ }^{1}$, Joel D. Brown, M.D., Colonel, MC, USA ${ }^{2}$, and John W. Evans, Jr., M.D., Major, MC, USA ${ }^{3}$

${ }^{1}$ Department of Radiology, Brooke Army Medical Center, Fort Sam, Houston, Texas, USA,

${ }^{2}$ Department of Medicine, and ${ }^{3}$ Department of Radiology, Tripler Army Medical Center, Honolulu, Hawaif, USA

An inadvertent and unwitting typographical error was printed in the Diagnosis recorded at the top of page 224. The Diagnosis should read:

Myonecrosis of Left Leg Simulating Gas Gangrene Due to Gram-Negative Organisms.

Sincere apologies are extended to the authors for this inadvertent mistake. 